\title{
Results of surgical therapy in patients with adenocarcinoma of the esophagus and cardia
}

\author{
Alberto Peracchia, Luigi Bonavina, Raffaello Incarbone, and Antonio Floridi \\ Department of General Surgery and Surgical Oncology, University of Milan, Ospedale Maggiore Policlinico I.R.C.C.S., Milan, Italy
}

\begin{abstract}
:
Background. Adenocarcinoma of the esophagus and cardia is a challenging disease for the surgeon. Delay in diagnosis, nodal involvement, and incompleteness of resection have an adverse effect on long-term prognosis. Efforts are currently oriented to identify patients who may benefit from extensive resection.

Methods. Between November 1992 and May 1998, 218 patients with histologically proven adenocarcinoma of the distal esophagus or cardia were referred to our Department. In 6 patients $(\mathbf{1 0 . 2} \%)$ cancer was discovered during endoscopic surveillance for Barrett's metaplasia. Overall, 147 patients (67\%) underwent resection. An Ivor-Lewis approach was used in 121 patients; of these, 51 underwent an extended mediastinal lymph node dissection.

Results. Median cumulative survival was $25.9 \pm 3.1$ months in patients undergoing resection, and $7 \pm 1.3$ months in patients having palliation $(P<0.01)$. Survival was significantly higher in patients with negative nodes than in those with lymph node metastases $(54 \pm 12.9$ versus $17 \pm 2.8$ months; $P<0.01)$. Six of the 51 patients $(11.8 \%)$ undergoing extended lymphadenectomy had metastatic upper mediastinal nodes. Additional serial sections and immunohistochemistry were performed in 46 patients. In 6 of 18 patients $(33.3 \%)$ with negative nodes at conventional hematoxylin-eosin examination, immunohistochemistry demonstrated micrometastases in the lesser curvature, paracardial, peripancreatic, or lower mediastinal nodes. Three of these patients had recurrent disease within the first year of follow-up.

Conclusions. Early diagnosis remains the prerequisite for curative treatment of adenocarcinoma of the esophagus and cardia. Endoscopic surveillance appears to be warranted in patients with Barrett's metaplasia. When a curative resection is attempted, extended lymphadenectomy improves tumor staging and may prevent local recurrences. Serial sections and immunohistochemistry provide additional accuracy in the staging of the disease and may prove useful to select patients for adjuvant therapy.
\end{abstract}

Offprint requests to: A. Peracchia

Received for publication on Sept. 3, 1998; accepted on March 3, 1999
Key words: adenocarcinoma, Barrett's metaplasia, cardia, esophagus, lymphadenectomy, micrometastases, neoadjuvant chemotherapy

\section{Introduction}

It has been shown that the incidence of esophageal adenocarcinoma is increasing at an alarming rate in Western societies [1]. Barrett's metaplasia has been reported to be associated with a higher risk of development of adenocarcinoma of the distal esophagus [2]; its link with a long-lasting history of gastroesophageal reflux disease underscores the importance of including patients with Barrett's esophagus in a surveillance program for early diagnosis of malignancy [3]. Although surgical resection of the tumor remains the mainstay of treatment, controversy still exists about the extent of the esophagogastric resection, the extent of lymphadenectomy, and the possible role of neoadjuvant therapies. The purpose of this study was to report our experience in patients with adenocarcinoma of the distal esophagus and cardia observed and treated at a single institution over the past 6 years.

\section{Patients and methods}

Between November 1992 and May 1998, 218 patients with histologically proven adenocarcinoma of the distal esophagus or esophagogastric junction were referred to our Department. According to Siewert's classification [4] there were 59 patients with type I tumor (Barrett's adenocarcinoma), 127 with type II (true cardia adenocarcinoma), and 32 with type III (subcardial adenocarcinoma). Demographic data and clinical history were recorded for each patient. Preoperative diagnostic assessment included: barium swallow study, chest X-ray, upper gastrointestinal 
endoscopy with lugol staining-guided biopsy, thoracoabdominal computed tomography (CT) scan, and cervical and abdominal ultrasonography (US). Endoscopic ultrasonography (EUS) was carried out in patients with doubtful peritumoral cleavage planes or as an alternative to CT scan. Preoperative laparoscopy with laparoscopic ultrasonography was performed in patients with locally advanced tumors (T3/T4) in order to exclude abdominal spread, i.e., peritoneal carcinosis and small superficial liver metastases.

The operation was carried out through an upper midline laparotomy followed by a right postero-lateral thoracotomy (Ivor-Lewis approach) in the majority of the patients. A combined cervical and abdominal approach with video-assisted trans-mediastinal dissection was reserved for patients with anesthesiologic contraindications to thoracotomy or with Barrett's esophagus when the metaplasia reached the proximal esophagus. In patients with cancer developing from the gastric side of the esophagogastric junction (type III), a total gastrectomy was generally performed through a midline laparotomy. In patients undergoing surgery with curative intent, an abdominal D2 lymph node dissection was routinely performed. In the chest, lymphadenectomy included the periesophageal, subcarinal, and parabronchial nodes. During the past 3 years, upper mediastinal nodes were also resected. The thoracic duct was routinely ligated just above the diaphragm. In patients undergoing an Ivor-Lewis or a trans-mediastinal approach, the stomach was used as an esophageal substitute in all but one patient, who had previously undergone esophagogastric resection for high-grade dysplasia on Barrett's esophagus through a left thoracotomy. In patients undergoing total gastrectomy, reconstruction with a Roux en-Y jejunal loop was used. The esophagogastric and esophagojejunal anastomosis were usually performed with a circular stapler (CEEA or PCCEA 25; USSC, Norwalk, $\mathrm{CT}$, USA) above the ARCH of the azygos vein and at the level of the inferior pulmonary vein, respectively.
After a trans-mediastinal esophagectomy, an esophagogastric anastomosis was performed in the neck in one layer, using a running suture (Maxon 4-0; Davis \& Geck, American Cyanamid, Wayne, NJ, USA).

Patients with doubtful cleavage planes at preoperative investigation underwent neoadjuvant therapy with cisplatin (CDDP) and 5-fluorouracil (5FU), or the CDDP, 5FU, leucovorin and etoposide (PELF) regimen. Non-operable patients and those with distant metastases underwent endoscopic palliation. This consisted of laser therapy or alcohol injection for vegetant lesions, and intubation for infiltrating tumors. Whenever possible, adjuvant chemotherapy was administered.

The tumors were staged according to the guidelines of the International Union against Cancer (UICC) (1997) [5]. Type I and II tumors were classified as esophageal cancer; type III tumors as gastric cancer. Additional serial sections and immunohistochemistry were performed to assess whether the rate of metastatic lymph nodes detected in the surgical specimen could increase. Additional sections of all paraffin blocks, 3- $-\mu \mathrm{m}$ in thickness, were obtained, and two adjacent sections were stained with hematoxylin-eosin and anticytokeratin monoclonal antibodies (MoAbs) (AE1 and AE3 MoAbs, Signet Laboratories, Dedham, MA, USA).

The Mann-Whitney $U$ and $\chi^{2}$ tests were used for comparison of groups. The Kaplan-Meier method and log-rank test were used for evaluation of survival. Values were reported as means \pm SEM, unless otherwise stated. Statistical significance was taken for $P<0.05$.

\section{Results}

The demographic data and features of patients with adenocarcinoma compared with those with squamouscell carcinoma of the thoracic esophagus observed during the same period are listed in Table 1. The demographic data and features of patients with

Table 1. Characteristics of patients with adenocarcinoma of the gastroesophageal junction (ACA) compared with those of patients with squamous-cell carcinoma of the thoracic esophagus (SCC)

\begin{tabular}{lccc}
\hline & ACA $(n=218)$ & SCC $(n=280)$ & $P$ \\
\hline Age (years) & 62 (range, $19-91)$ & $61($ range, $29-97)$ & NS \\
Sex ratio M:F & $4.9: 1$ & $3.5: 1$ & NS \\
Height $(\mathrm{m})$ & $1.70 \pm 0.006$ & $1.68 \pm 0.005$ & $<0.01$ \\
Weight $(\mathrm{kg})$ & $69.7 \pm 0.9$ & $64.2 \pm 0.9$ & $<0.01$ \\
Body mass index & $24.0 \pm 3.2$ & $22.5 \pm 4.2$ & $<0.01$ \\
History of GERD & $36(16.5 \%)$ & $8(3 \%)$ & $<0.01$ \\
\hline
\end{tabular}

Observed between 1992 and 1998

GERD, Gastroesophageal reflux disease 
Table 2. Characteristics of patients according to type of adenocarcinoma [4]

\begin{tabular}{|c|c|c|c|c|}
\hline & Barrett's $(n=59)$ & Cardia $(n=127)$ & Subcardial $(n=32)$ & $P$ \\
\hline Age (years) & 59 (range, 19-89) & 63 (range, 31-91) & 64 (range, 24-82) & NS \\
\hline Sex ratio $M: F$ & $5.5: 1$ & $4.1: 1$ & $9.6: 1$ & NS \\
\hline Height (m) & $1.71 \pm 0.01$ & $1.70 \pm 0.008$ & $1.71 \pm 0.02$ & NS \\
\hline Weight (kg) & $70.6 \pm 1.9$ & $69.6 \pm 1.2$ & $70.3 \pm 2.2$ & NS \\
\hline Body mass index & $23.7 \pm 0.5$ & $24.1 \pm 0.3$ & $23.9 \pm 0.5$ & NS \\
\hline History of GERD & $23(39 \%)$ & $10(7.8 \%)$ & $3(9.4 \%)$ & $<0.01 *$ \\
\hline Resected & $46(78 \%)$ & $83(65.3 \%)$ & $18(56.2 \%)$ & $<0.05 *$ \\
\hline pTNM Stage 0-I & $11(23.9 \%)$ & $7(8.4 \%)$ & $2(11.1 \%)$ & $<0.01 * *$ \\
\hline $\mathrm{N}+$ patients & $25(54.3 \%)$ & $56(67.5 \%)$ & $14(77.8 \%)$ & NS \\
\hline $\mathrm{R} 0$ resection & $42(91.3 \%)$ & $65(78.3 \%)$ & $14(77.8 \%)$ & $<0.01^{*}$ \\
\hline
\end{tabular}

*Barrett's versus cardia and subcardial carcinoma; **Barrett's versus cardia cancer. GERD, Gastroesophageal reflux disease, R0, Complete resection

esophagogastric adenocarcinoma classified by type according to Siewert's classification [4] are reported in Table 2. Three patients were referred to our Department after a previous misdiagnosis of esophageal achalasia: one had been treated with intrasphincteric botulinum toxin injection 1 year earlier, and one with endoscopic dilation. In six patients (10.2\%) with Barrett's adenocarcinoma, cancer was discovered during endoscopic surveillance for Barrett's esophagus; of these, one had had Nd:YAG laser ablation of metaplastic epithelium (intestinal metaplasia without dysplasia) and another had undergone esophagogastric resection through a left thoracotomy. Of these six patients, one had high-grade dysplasia and three had stage I adenocarcinoma. Conversely, among the nonsurveyed patients with Barrett's esophagus, there were seven early-stage tumors $(P<0.01)$.

Fifteen patients underwent up to five cycles of neoadjuvant chemotherapy (median, two); in 2 patients the treatment was stopped due to hematologic or gastrointestinal intolerance. All individuals were resected, and none had a complete pathological response. Resection was considered complete (R0) in 10 patients, and R1 (microscopic residual tumor) in 5. Perioperative morbidity was not increased in these patients compared with those who did not have neoadjuvant therapy.

Preoperative laparoscopy was performed at the same session as the planned surical resection in 45 patients. It led to a change in the therapeutic approach in 5 patients (11\%): 3 patients with peritoneal carcinosis and 1 patient with a superficial metastasis of segment III undetected at preoperative CT scan and US did not undergo the planned resection; conversely, 1 patient with a liver hemangioma simulating a metastatic mass had resection.

One hundred forty-seven patients (67\%) underwent resection. An Ivor-Lewis approach (laparotomy and right thoracotomy) was used in 121 patients; of these, 51 underwent extended mediastinal lymph node dissec-
Table 3. Prevalence of patients with positive nodes in relation to tumor wall penetration

\begin{tabular}{lcc}
\hline T status & Patients $(n)$ & Node positive $(\%)$ \\
\hline Tis & 2 & $0(0)$ \\
T1 & 18 & $3(16.6)$ \\
T2 & 28 & $16(57.1)$ \\
T3 & 88 & $73(82.9)$ \\
T4 & 11 & $9(81.8)$ \\
\hline
\end{tabular}

tion. A video-assisted trans-mediastinal esophagectomy was performed in 14 patients. Twelve patients underwent total gastrectomy. Overall, postoperative mortality was $2 \%$ and included 2 patients with a leak of the intrathoracic esophagogastric anastomosis, and 1 with pulmonary embolism. The prevalence of patients with positive nodes increased proportionally in relation to the wall penetration of tumor (Table 3 ). The cumulative survival according to Siewert's type of tumor [4] is reported in Fig. 1. The median overall survival of patients undergoing resection $(n=147)$ was $26 \pm 3.9$ months (95\% confidence intervals [CI], 18.2-33.8), whereas the median survival of those having endoscopic palliation $(n=71)$ was $7 \pm 1.35$ months $(95 \%$ CI, $4.7-$ 9.6).

Survival was significantly higher in patients with negative nodes than in those with lymph node metastases $(54 \pm 12.9$; 95\% CI, 28.7-79.2 versus $17 \pm$ 2.8; 95\% CI, 11.4-22.6 months; $P<0.01$ ); all patients with negative nodes had a complete resection (R0). Survival was significantly increased in patients undergoing a complete resection compared with those with R1 and R2 (macroscopic residual tumor) (Fig. 2). No survival advantage was noted in patients who had previous neoadjuvant treatment. Survival by stage in patients with type I and II tumors is reported in Fig. 3. Postoperative staging and survival by stage in patients with subcardial (type III) cancer are listed in Table 4. 


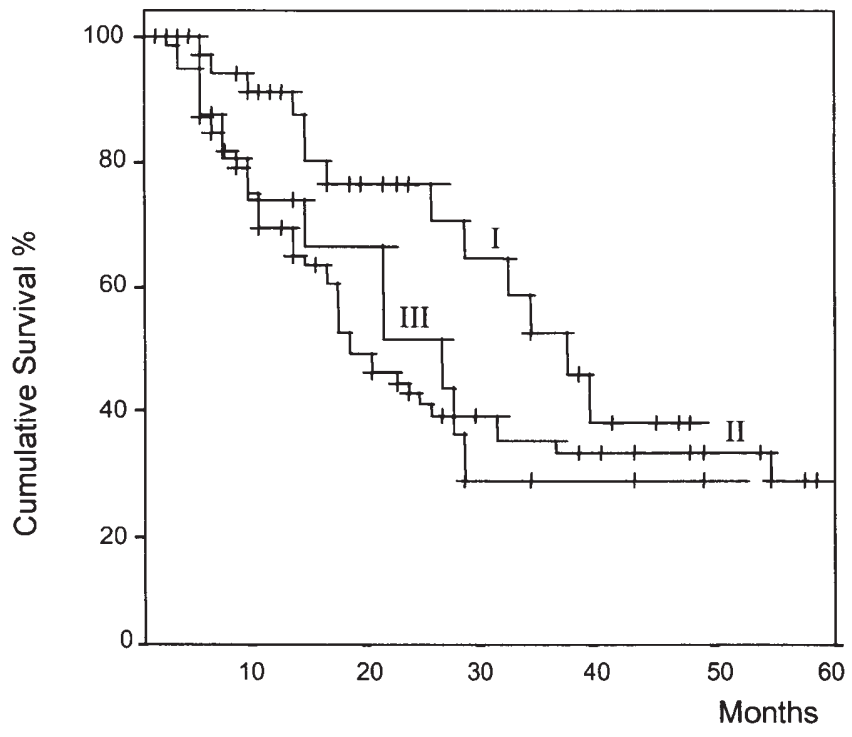

Fig. 1. Cumulative survival of patients with Barrett's (type $I$ ), cardia (type $I I$ ), and subcardial (type $I I I$ ) adenocarcinoma. Log rank test, $P=0.18$

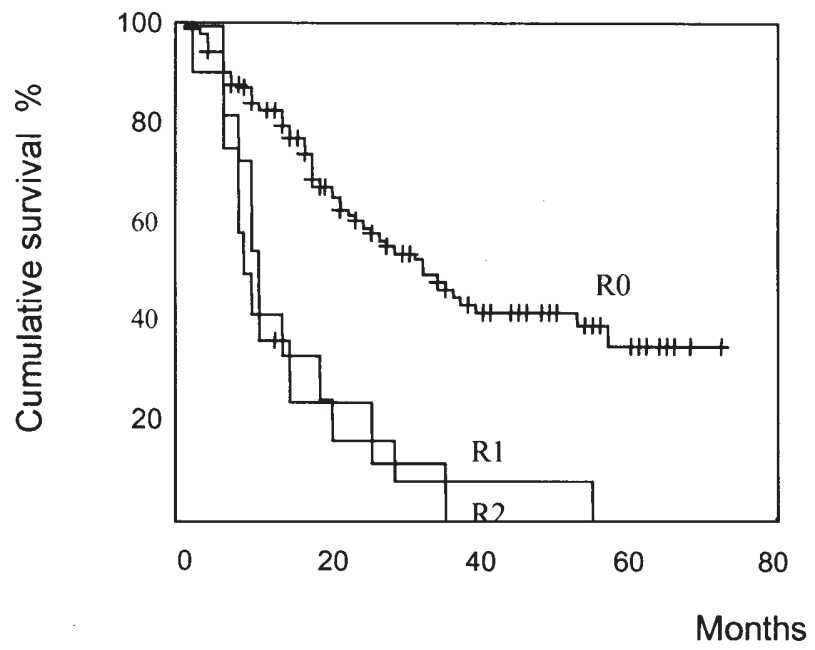

Fig. 2. Cumulative survival of patients undergoing a complete resection $(R O)$ compared with those with microscopic $(R 1)$ or macroscopic $(R 2)$ residual tumor. Log rank test, $P<0.01$

Six of the 51 patients (11.8\%) undergoing extended lymphadenectomy had metastatic upper mediastinal nodes (Table 5). Five of these individuals had a Siewert type I tumor.

Additional serial sections and immunohistochemistry were performed in 46 patients. In 6 of 18 patients $(33.3 \%)$ with negative nodes at conventional hematoxylin-eosin examination, immunohistochemistry demonstrated micrometastases in 18 lymph nodes, including lesser curvature, paracardial, peripancreatic, and lower mediastinal nodes. In contrast to the indi-

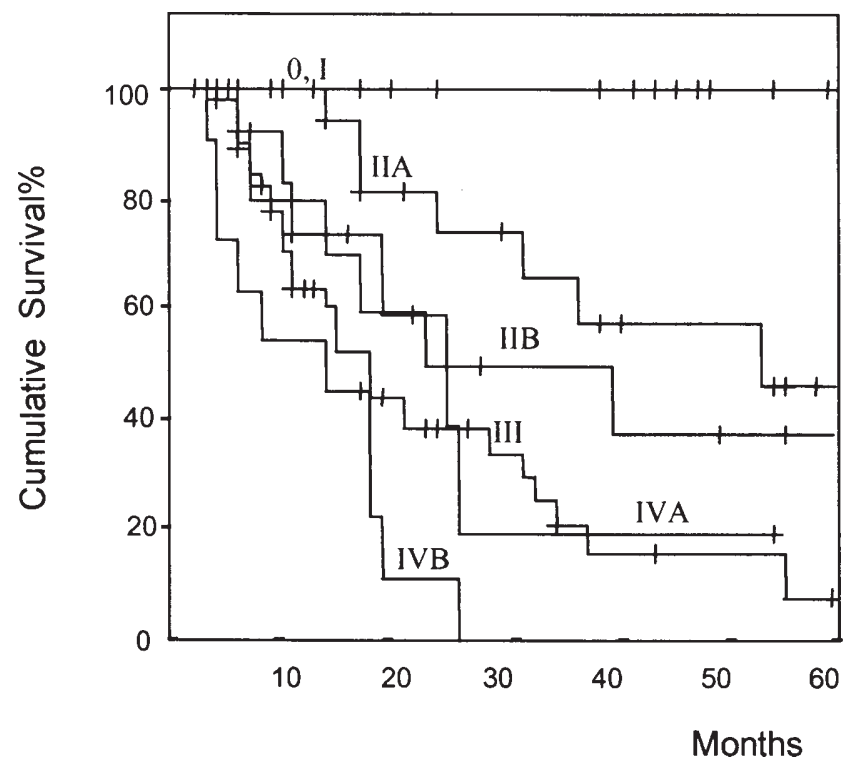

Fig. 3. Cumulative survival of patients with adenocarcinoma of the distal esophagus and cardia (type I and II tumors) according to tumor stage

Table 4. Staging and survival of patients with subcardial (type III) adenocarcinoma

\begin{tabular}{lcccc}
\hline Stage & Patients & $\begin{array}{c}\text { Mean survival } \\
\text { (months) }\end{array}$ & SE & $95 \%$ CI \\
\hline IA & 1 & Alive at 27 months & - & - \\
IB & 1 & Alive at 6 months & - & - \\
II & 2 & 35 & 9.9 & $15.6-54.4$ \\
IIIA & 6 & 29.8 & 5.2 & $19.6-40.0$ \\
IIIB & 3 & 23 & 6.0 & $11.2-34.8$ \\
IV & 5 & 5.7 & 0.7 & $4.4-7.0$ \\
\hline
\end{tabular}

CI, Confidence intervals

viduals confirmed N0 at cytokeratyn analysis, all of whom were alive and disease-free, half of the patients with nodal micrometastases had recurrent disease within a year. Moreover, in 18 of 28 patients (64.3\%) with metastatic nodes at conventional staining, serial sections and immunohistochemistry showed additional positive nodes.

\section{Discussion}

With the rising incidence of Barrett's esophagus in Western countries, the ratio between adenocarcinoma of the lower esophagus and squamous-cell carcinoma has changed markedly over the past decade [6,7]. It has to be emphasized that most tumors of the cardia arise from short segments of Barrett's esophagus and should therefore, according to some investigators, be considered esophageal tumors $[2,8]$. Patients with Barrett's 
Table 5. Complication rates, pattern of recurrent disease, and median cumulative survival according to the extent of mediastinal lymphadenectomy in patients undergoing trans-thoracic esophagectomy

\begin{tabular}{lcc}
\hline & $\begin{array}{c}\text { Extended }(n=51) \\
(\%)\end{array}$ & $\begin{array}{c}\text { Standard }(n=70) \\
(\%)\end{array}$ \\
\hline Mortality & $1(1.9)$ & $2(2.9)$ \\
Morbidity & $5(9.8)$ & $15(21.4)$ \\
$\quad$ Leak & $2(3.9)$ & $8(11.4)$ \\
Pulmonary & $3(5.9)$ & $4(5.7)$ \\
Gastric tube necrosis & - & $1(1.9)$ \\
Gastrobronchial fistula & - & $1(1.4)$ \\
$\quad$ Chylothorax & - & $1(1.4)$ \\
Recurrence & $2(3.9)$ & $10(14.2)$ \\
$\quad$ Mediastinal & $16(31.3)$ & $18(25.6)$ \\
Systemic & & $24.9(6.6-41.4)^{*}$ \\
Median cumulative survival, & & \\
$\quad$ months (95\% confidence & $4.8(22.6-41.4)$ & \\
$\quad$ interval) & & \\
\hline
\end{tabular}

* $P$ by log rank test $=0.26$

metaplasia have an increased risk of 30-125 times that in the normal population of developing esophageal adenocarcinoma [9]. It has been shown that patients under an endoscopic surveillance program for Barrett's esophagus have the malignancy detected at an earlier stage and have a better prognosis than patients with newly recognized Barrett's adenocarcinoma [10]. In our series, four of six endoscopically surveyed patients were operated on at an early stage. The prevalence of gastroesophageal reflux disease and the body mass index were significantly higher in patients with adenocarcinoma than in the group with squamous-cell carcinoma. The prevalence of reflux disease was also significantly higher in the individuals with Barrett's carcinoma than in the other groups.

It has been reported that computed tomography and transabdominal ultrasonography correctly stage only $64 \%$ of patients with cancer of the lower esophagus [11]. Preoperative laparoscopy may add significant findings and lead to changes, in the therapeutic approach in $10 \%-20 \%$ of patients [11,12]. In our study, laparoscopy led to a change in the therapeutic strategy in $11 \%$ of the patients.

Cancer of the lower esophagus and cardia, when treated at an early stage, should be regarded today as a potentially curable disease [13-15]. Analyzing the results of surgery according to the histological type, Streitz et al. [16] showed that the 5-year survival rate after resection was $23.7 \%$ for patients with Barrett's adenocarcinoma, $22.4 \%$ for patients with cardia adenocarcinoma, and $17.3 \%$ for those with squamouscell carcinoma; no statistically significant difference between these groups was found. In our series, there was no significant difference in terms of long-term survival for patients with type I, II, or III tumors. Cumulative survival was higher in patients with negative nodes compared with findings in those with metastatic nodes. Furthermore, it should be noted that all patients with negative nodes had had a complete resection (R0).

Although surgical resection remains the standard treatment of cancer of the esophagus and cardia, the role and the optimal extent of lymphadenectomy are still controversial issues. Since an R0 resection represents a critical prognostic factor, it appears that lymphadenectomy of regional nodes must be radical. However, the type of lymphadenectomy is far from being unanimously defined. An anatomical classification of the extent of lymphadenectomy for cancer of the thoracic esophagus has recently been reported [17]. The abdominal compartment (field 1) encompasses all nodal stations limited inferiorly by the pancreas, superiorly by the esophageal hiatus, and laterally by the splenic hilum and the hepatoduodenal ligament. Removal of additional lymph nodes (stations 13 and 16) and the technique of pancreas-preserving lymphadenectomy of the splenic vessels may be required in type III tumors $[18,19]$. The mediastinal compartment (field 2) includes three different levels of lymphadenectomy: "standard", i.e., removal of paraesophageal, subcarinal, and right parabronchial nodes; "extended" (2-field one), i.e., removal of right paratracheal, brachiocephalic, and right recurrential nodes; "total" (2-field two), i.e., removal also of the left recurrential nodes. The cervical compartment (field 3) includes the lymphatic tissue in the neck with sparing of the sternocleidomastoid muscle and the jugular vein. In our series, 6 of 51 patients $(11.8 \%)$ undergoing extended lymphadenectomy had upper mediastinal metastatic nodes. This more extensive nodal dissection did not increase the postoperative morbidity 
rate, as reflected by the low incidence of recurrent nerve palsy $(1.4 \%)$ and respiratory complications $(4.8 \%)$.

Previous studies in patients with breast [20], colon [21], and gastric cancer [22] have shown the benefits of routine detection of occult micrometastases in regional lymph nodes. Based on the results of such studies, which demonstrate the frequent occurrence of micrometastases, some authors have advocated a wide prophylactic lymph node dissection [23]. In our experience, serial sections and immunohistochemistry allowed us to stage as N1 one-third of patients previously staged as $\mathrm{N} 0$ at standard histological examination, and allowed us to discover new positive nodes in two-thirds of patients staged as $\mathrm{N} 1$ at conventional staining. At present, no inference can be made regarding the potential benefits of an extended lymphadenectomy in patients with occult nodal metastases. Conversely, the upstaging of the disease obtained by detection of nodal micrometastases may be useful in selecting patients for adjuvant therapy.

In conclusion, early diagnosis remains the prerequisite for curative treatment of adenocarcinoma of the esophagus and cardia. Endoscopic surveillance appears to be warranted in patients with Barrett's metaplasia. The role of neoadjuvant therapy is still uncertain. When a curative resection is attempted, extended lymphadenectomy allows precise tumor staging and may decrease the incidence of local recurrences. However, the potential benefits of an extended lymphadenectomy should be tested in prospective randomized clinical studies.

Acknowledgments. This work was supported by F.I.R.C. (Fondazione Italiana per la Ricerca sul Cancro)

\section{References}

1. Thomas P, Doddoli C, Lienne N, Morati N, et al. Changing patterns and surgical results in adenocarcinoma of the oesophagus. Br J Surg 1997;84:119-25.

2. Clark GWB, Smyrk TC, Burdiles P, et al. Is Barrett metaplasia the source of adenocarcinomas of the cardia? Arch Surg 1994;129:609-14.

3. DeMeester TR, Attwood SE, Smirk TC, Therkildsen DH, Hinder RA. Surgical therapy in Barrett's esophagus. Ann Surg 1990;212:528-42.

4. Siewert JR, Stein HJ. Carcinoma of the gastroesophageal junction - classification, pathology and extent of resection. Dis Esoph 1996;9:173-82.
5. UICC. TNM classification of malignant tumors. Jobin L, Wittekind C, editors. 5th Ed.: Wiley-Liss, 1997.

6. Pera M, Cameron A, Trastek V, Carpenter H, Zinsmeister A. Increasing incidence of adenocarcinoma of the esophagus and esophagogastric junction. Gastroenterology 1993;104: 510-3.

7. Blot W, Devesa S, Fraumeni J. Continuing climb in rates of esophageal adenocarcinoma [letter]. JAMA 1993;270:1320.

8. Hamilton S, Smith R, Cameron J. Prevalence and characteristic of Barrett esophagus in patients with adenocarcinoma of the esophagus and esophagogastric junction. Hum Pathol 1988;19: 942-8.

9. Van Der Veen AH, Dees J, Blankenstein JD, Blankenstein MV. Adenocarcinoma in Barrett's esophagus: an overrated risk. Gut 1989;30:14-8.

10. Peters JH, Clark GWB, Ireland AP, Chandrasoma P, Smyrk TC, DeMeester TR. Outcome of adenocarcinoma arising in Barrett's esophagus in endoscopically surveyed and nonsurveyed patients. J Thorac Cardiovasc Surg 1994;108:813-22.

11. Anderson D, Campbell S, Park K. Accuracy of laparoscopic ultrasonography in the staging of upper gastrointestinal malignancy. Br J Surg 1996;83:1424-8.

12. Bonavina L, Incarbone R, Lattuada E, Cesana B, Peracchia A. Preoperative laparoscopy in management of patients with carcinoma of the esophagus and of the esophagogastric junction. $\mathbf{J}$ Surg Oncol 1997;65:171-4.

13. Holscher AH, Elfriede E, Bumm R, Bartels H, Hofler H, Siewert JR. Prognostic factors of resected adenocarcinoma of the esophagus. Surgery 1995;118:845-55.

14. Hagen J, Peters J, De Meester T, et al. Superiority of extended en bloc esophagogastrectomy for carcinoma of the lower esophagus and cardia. J Thorac Cardiovasc Surg 1993;106:850-9.

15. Peracchia A, Bonavina L, Incarbone R, Chella B. Intérét de la lymphadenectomie étendue dans le cancer du bas oesophage et du cardia. J Chir 1997;134:209-13.

16. Streitz J, Ellis F, Gibb S, Balogh K, Watkins E. Adenocarcinoma in Barrett's esophagus: a clinico-pathologic study of 65 cases. Ann Surg 1991;213:122-5.

17. Bumm R, Wong J. Extent of lymphadenectomy in esophagectomy for squamous-cell carcinoma: How much is necessary? Dis Esoph 1994;7:151-5.

18. Maruyama K, Gunven P, Okabayasha K, Sasako M, Kinoshita T. Lymph node metastases of gastric cancer. Ann Surg 1989;210: 596-602.

19. Maruyama K, Sasako M, Kinoshita T, Okajima K. Effectiveness of systematic lymph node dissection in gastric cancer surgery. In: Nishi M, Ichikawa H, Nakajima T, Maruyama K, Tahara E, editors. Gastric cancer. Berlin Heidelberg New York Tokyo: Springer, 1993:293-305.

20. Noguchi S, Aihara T, Nakamory S, et al. The detection of breast carcinoma micrometastases in axillary lymph nodes by means of reverse transcriptase polymerase chain reaction. Cancer 1994;74: 1595-600.

21. Greenson J, Isenhart C, Rice, et al. Identification of occult micrometastases in pericolic lymph nodes of Dukes' B colorectal cancer patients using monoclonal antibodies against cytokeratin and CC49. Cancer 1994;73:563-9.

22. Maehara Y, Oshiro T, Baba H, et al. Clinical significance of occult micrometastasis in lymph nodes from patients with early gastric cancer who died of recurrency. Surgery 1996;119:397-402.

23. Siewert J, Kestlemeier R, Busch R, et al. Benefits of D-2 lymph node dissection for patients with gastric cancer and pN0 and pN1 lymph node metastases. Br J Surg 1996;83:1144-7. 